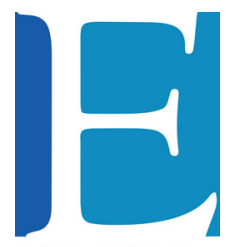

2018, Vol. 18, No 2.

ISSN 1667-4545

Recuperado de https://revistas.unc.edu.ar/index.php/revaluar

Laboratorio de Evaluación Psicológica y Educativa

EVALUAR

Facultad de Psicología - Universidad Nacional de Córdoba

\title{
Revisión de metodologías empleadas en los estudios factoriales de la Escala de Bienestar Psicológico de Ryff (versión en español)
}

\section{Review of Methodologies Used in Factorial Studies of Ryff's Psychological Well- being Scales (Spanish Version)}

\author{
Sergio Dominguez-Lara * 1 , Jhonatan S. Navarro-Loli ${ }^{1}$ \\ 1 - Instituto de Investigación de Psicología, Universidad de San Martín de Porres, Lima, Perú.
}

\author{
Introducción \\ Método \\ Resultados \\ Discusión \\ Referencias
}

Recibido: 29/11/2017 Revisado: 06/01/2018 Aceptado: 29/01/2018

\section{Resumen}

El objetivo de esta investigación fue realizar una revisión de la literatura para describir la metodología utilizada en estudios factoriales de la versión en español de la Escala de Bienestar Psicológico de Ryff (PWBS-E; Diaz et al., 2006). Se efectuó una búsqueda directa en bases de datos de estudios instrumentales que analizan la PWBS-E y se encontraron nueve artículos con información relevante. En cuanto a los estudios que emplean análisis factorial confirmatorio, se observaron diferencias respecto al software utilizado y a los métodos de estimación, pero la mayoría no indica el tipo de matriz de correlaciones utilizada. Del mismo modo, el grado en que se informan correlaciones interfactoriales y cargas factoriales es bajo y, en promedio, los índices de ajuste son menores a los límites aceptables. Dos de los tres estudios bajo un enfoque exploratorio destacan por el uso de análisis de componentes principales y rotación varimax, mientras que solo uno informa las cargas factoriales. De acuerdo a los resultados obtenidos, el uso de la puntuación total de la PWBS-E no está justificado. Se concluye que hay una falta de claridad con respecto a la estructura interna de la PWBS-E.

Palabras clave: bienestar, Escala de Bienestar Psicológico de Ryff, análisis factorial, revisión

\section{Abstract}

The aim of this investigation was to perform a literary review describing the methodology used in factorial studies of the Ryff's Psychological Well-Being Scales - Spanish Version (PWBS-E; Diaz et al., 2006). A direct search of digital databases for instrumental studies that analyze the PWBS-E identified nine papers providing relevant information. As for studies using confirmatory factor analysis, differences regarding software and estimation methods were observed, but the majority of them does not indicate which type of correlation matrix was used. Likewise, there is little reported on inter-factor correlations and factor loadings, and the fit indices are lower than the acceptable boundaries. Two out of three exploratory factor analysis studies use the principal components analysis and varimax rotation, while only one reports factor loadings. In general, the use of the PWBS-E total score is not justified. The study concludes about the lack of clarity of the PWBS-E internal structure.

Keywords: well-being, Ryff's Psychological Well-Being Scale, factor analysis, review

*Correspondencia a: Sergio Dominguez Lara. Instituto de Investigación de Psicología, Universidad de San Martín de Porres, Av. Tomás Marsano 242 (5to piso), Lima 34 - Perú. Correo electrónico: sdominguezmpcs@gmail.com.

Cómo citar este artículo: Dominguez-Lara, S., \& Navarro-Loli, J. S. (2018). Revisión de metodologías empleadas en los estudios factoriales de la Escala de Bienestar Psicológico de Ryff (versión en español). Revista Evaluar, 18(2), 17-30. Recuperado de https://revistas.unc.edu.ar/index.php/revaluar 


\section{Introducción}

El modelo de Bienestar Psicológico (BP) propuesto por Ryff $(1989,1995)$ ha tenido un impacto significativo en el mundo académico. Este planteamiento se basa en la concepción eudaemónica del bienestar, es decir, resalta aspectos vinculados con el potencial humano y el significado de la vida (Chen, Jing, Hayes, \& Lee, 2013). Concretamente, se basa en la forma como el individuo afronta los retos y su rol en el crecimiento personal, así como en la forma de establecer relaciones armoniosas con los demás. En consecuencia, el instrumento derivado de estos planteamientos, la Psychologica WellBeing Scale (PWBS; Ryff \& Keyes, 1995), es uno de los más empleados a nivel mundial tanto en investigación básica como aplicada, dado el creciente interés en este tópico del conocimiento psicológico (Pérez-Verduzco \& Laca-Arocena, 2017). La PWBS está compuesta por seis dimensiones: autoaceptación (AA), relaciones positivas con los otros (RPO), autonomía (AU), dominio del entorno (DE), propósito en la vida (PV) y crecimiento personal (CP); de los cuales, según las referencias originales, también es posible obtener una puntuación total. La PWBS es uno de los instrumentos más usados a nivel mundial (Abbott, Ploubidis, Huppert, Kuh, \& Croudace, 2010); y por lo tanto, considerando su amplia difusión en el ámbito académico, es necesario que cumpla con algunos estándares para su utilización, como los propuestos por The Standards for Educational and Psychological Testing (AERA, APA, \& NCME, 2014), y de metodología psicométrica recomendada actualmente.

El modelo de Ryff postula al BP como un constructo de orden superior, cuyas dimensiones se encuentran teóricamente diferenciadas. De este modo, la correspondencia del BP con la PWBS implicaría que la correlación entre dimensiones es lo suficientemente elevada para indicar que existe relación empírica, pero no tanto como para sugerir multicolinealidad o solapamiento factorial. Por el contrario, una correlación de magnitud elevada $(r>.80)$ sugeriría redundancia entre factores así como la posibilidad de que exista un factor general (FG) capaz de explicar esa variabilidad. Del mismo modo, y asociado a la idea anterior, al ser posible obtener e interpretar una puntuación total de la PWBS como se indicó anteriormente, es necesario que exista evidencia de la fortaleza de un FG denominado BP que pueda explicar satisfactoriamente la variabilidad de los ítems.

La PWBS ha sido traducida a múltiples idiomas, en español se cuenta con las versiones de Villar, Osuna, Solé y Triadó (2005); Triadó, Villar, Solé y Celdrán (2007) y con la de Díaz et al. (2006), cuya estructura factorial es similar a la de la original (Ryff \& Keyes, 1995), aunque con 10 ítems menos. Esta versión ha sido usada hasta la fecha en múltiples investigaciones para la evaluación del BP. La versión de Díaz et al. (2006), en comparación con las de Villar et al. (2005) y Triadó et al. (2007), cuenta con estudios instrumentales realizados en países de habla hispana en los que se evaluaron sus propiedades psicométricas mediante un enfoque analítico factorial a fin de obtener evidencias dentro del marco actual de validez (Ríos \& Wells, 2014), mediante estrategias confirmatorias o exploratorias (Ríos \& Wells, 2014) y mediante modelos bifactor (Canivez, 2016; Reise, 2012), aunque con resultados heterogéneos respecto a diversos indicadores psicométricos relevantes para dichos métodos.

Por ello, el objetivo del presente estudio fue realizar una revisión de estudios instrumentales a fin de describir la metodología utilizada para analizar la dimensionalidad de la PWBS-E en 
investigaciones de corte psicométrico realizadas con la versión de Díaz et al. (2006).

\section{Método}

Diseño

Se trata de una revisión de las características de los estudios analítico-factoriales llevados a cabo con la adaptación española del PWBS (PWBS-E; Díaz et al., 2006).

\section{Criterios de inclusión}

Se consideraron estudios instrumentales en idioma inglés y español (Ato, López, \& Benavente, 2013) que analizan la estructura interna de la adaptación al español del PWBS de Díaz et al. (2006). No se establecieron restricciones con respecto al año de publicación, ubicación geográfica, ni tipo de muestra evaluada.

\section{Búsqueda de literatura}

La búsqueda se realizó durante el mes de Octubre del año 2017 en la base de datos de SCOPUS y en un motor de búsqueda general (Google Scholar). Se utilizaron como palabras clave propiedades psicométricas, psychometrics properties, validez, validity, Escala de Bienestar Psicológico Ryff, Psychological Well-being Scales Ryff, Díaz; aludiendo a los procedimientos para realizar el análisis de la dimensionalidad, nombre del instrumento y al autor de la adaptación española.

\section{Información por considerar}

En el marco de las revisiones sistemáticas y estudios metaanalíticos existen guías especializadas que brindan criterios orientativos para recopilar información, como el COSMIN (Mokkink et al., 2012), pero estos están orientados a aspectos generales de cada sección del artículo y no abarcan las particularidades de las estrategias de análisis factorial. En este sentido, se utilizaron como referencia estudios destinados a las características de las aplicaciones del análisis factorial (Izquierdo, Olea, \& Abad, 2014; Jackson, Gillaspy, \& Purc-Stephenson, 2009).

De forma general, el proceso de revisión y codificación de los artículos seleccionados se realizó por los dos autores solicitando la colaboración de un investigador cuando existía algún tipo de discrepancia entre los revisores. De los artículos se consideró la nacionalidad de la muestra, grupo etario y tamaño muestral. Dado que se esperaba encontrar artículos con base tanto en análisis factorial exploratorio (AFE) como confirmatorio (AFC), los criterios para cada tipo fueron diferenciados. En relación con el AFC, se recolectó información sobre el programa estadístico usado, matriz de correlaciones (Pearson o Policóricas), método de estimación, índices de ajuste usados, magnitud de correlaciones interfactoriales, modelo jerárquico evaluado, eliminación de ítems y selección del mejor modelo. En cuanto al AFE, se recabó información sobre el programa estadístico usado, matriz de correlaciones (Pearson o Policóricas), análisis preliminares (KMO, test de Bartlett), método de estimación, criterio para determinar el número de factores, tipo de rotación, magnitud de correlaciones interfactoriales y eliminación de ítems. 


\section{Resultados}

Resultado de la búsqueda

Fueron hallados en SCOPUS un total de 123 estudios que citan la adaptación, mientras que la cifra de citaciones se elevó a 495 en Google Scholar. De estos, fueron seleccionados 9 manuscritos por cumplir con las especificaciones previas (estudio instrumental con base en la
PWBS-E de Díaz et al., 2006), de los cuales seis usaron únicamente $\mathrm{AFC}$, un estudio solo $\mathrm{AFE}$, mientras dos de ellos ejecutaron inicialmente AFE y posteriormente AFC.

Estudios bajo un enfoque confirmatorio

Referente a los estudios de enfoque confirmatorio, el software más utilizado en los estudios es el AMOS (37.5\%), mientras que el grupo etario más frecuente es el de adultos, y la

Tabla 1a

Caracterización de los estudios bajo un enfoque factorial confirmatorio.

\begin{tabular}{|c|c|c|c|c|c|c|c|c|c|c|c|c|c|c|c|c|c|}
\hline \multirow[b]{2}{*}{ Estudio } & \multirow[b]{2}{*}{ País } & \multicolumn{4}{|c|}{$\begin{array}{c}\text { Grupo etario (tamaño } \\
\text { muestral) }\end{array}$} & \multicolumn{5}{|c|}{ Programa estadístico } & \multicolumn{3}{|c|}{$\begin{array}{c}\text { Matriz de } \\
\text { correlaciones }\end{array}$} & \multicolumn{4}{|c|}{ Método de estimación } \\
\hline & & $\mathrm{AD}$ & UNIV & $\mathrm{ADU}$ & ADUM & AMOS & LISREL & EQS & Mplus & NI & Pearson & Policórica & NI & ML & ML-R & WLSMV & NI \\
\hline 1 & $\begin{array}{c}\text { España/ } \\
\text { Colombia }\end{array}$ & & & 919 & & & & & $\mathrm{x}$ & & & $\mathrm{x}$ & & & & $\mathrm{X}$ & \\
\hline 2 & Chile & 335 & & & & $\mathrm{x}$ & & & & & & & $\mathrm{x}$ & $\mathrm{x}$ & & & \\
\hline 3 & Chile & & 691 & & & & $\mathrm{x}$ & & & & & & $\mathrm{x}$ & $\mathrm{x}$ & & & \\
\hline 4 & México & & 447 & 256 & & & & & & $\mathrm{x}$ & & & $\mathrm{x}$ & & $\mathrm{X}$ & & \\
\hline 5 & Chile & & & 1346 & 300 & & & $\mathrm{x}$ & & & & & $\mathrm{x}$ & & & & $\mathrm{X}$ \\
\hline 6 & Argentina & & 465 & & & $\mathrm{x}$ & & & & & & & $\mathrm{x}$ & & & & $\mathrm{X}$ \\
\hline 7 & Chile & & & 300 & & & & $\mathrm{x}$ & & & & & $\mathrm{x}$ & & & & $\mathrm{X}$ \\
\hline 8 & España & & 701 & & & $\mathrm{X}$ & & & & & & & $\mathrm{x}$ & $\mathrm{X}$ & & & \\
\hline
\end{tabular}

Nota. 1 Van Dierendonck et al. (2008); 2. Gallardo-Cuadra y Moyano-Díaz (2012); 3. Véliz-Burgos (2012); 4. Medina-Calvillo, Gutiérrez-Hernández y Padrós-Blázquez (2013); 5. Vera-Villarroel, Urzúa, Silva, Pavez, y Celis-Atenas (2013); 6. Reyna, Delfino, y Mele (2013); 7. Chitgian-Urzúa et al. (2013); 8. Freire et al. (2017). AD: Adolescentes; UNIV: Universitarios; ADU: Adultos; ADUM; Adultos mayores; NI: No informa; ML: Máxima verosimilitud; ML-R: Máxima verosimilitud-robusto; WLSMV: Weighted Least Squares Mean and Variance adjusted.

Tabla 1b

Caracterización de los estudios bajo un enfoque factorial confirmatorio.

\begin{tabular}{|c|c|c|c|c|c|c|c|c|c|c|c|c|c|c|c|c|c|c|c|}
\hline \multirow[b]{2}{*}{ Estudio } & \multirow[b]{2}{*}{ País } & \multirow[b]{2}{*}{$\chi^{2}$} & \multicolumn{6}{|c|}{ Índices de ajuste (del mejor modelo) } & \multirow[b]{2}{*}{ SRMR } & \multicolumn{2}{|c|}{$\begin{array}{l}\text { Magnitud } \\
\text { de las cargas } \\
\text { factoriales }\end{array}$} & \multicolumn{3}{|c|}{$\begin{array}{c}\text { Magnitud } \\
\text { de las } \\
\text { correlaciones }\end{array}$} & \multicolumn{2}{|c|}{$\begin{array}{c}\text { Modelo } \\
\text { jerárquico }\end{array}$} & \multicolumn{2}{|c|}{$\begin{array}{c}\text { Elimina } \\
\text { ítems }\end{array}$} & \multirow[t]{2}{*}{$\begin{array}{l}\text { Mejor } \\
\text { modelo }\end{array}$} \\
\hline & & & SB- $\chi^{2}$ & CFI & NNFI & TLI & GFI & RMSEA & & Sí & NI & $\mathrm{VL}$ & PO & NI & $\mathrm{BIF}$ & $2 \mathrm{DO}$ & No & Sí & \\
\hline 1 & $\begin{array}{c}\text { España/ } \\
\text { Colombia }\end{array}$ & 1898.3 & & .820 & & .930 & & & .070 & & $\mathrm{X}$ & $\mathrm{x}$ & & & & $\mathrm{x}$ & & $\mathrm{X}$ & $\begin{array}{l}6 \mathrm{~F}+ \\
1 \mathrm{DO}\end{array}$ \\
\hline 2 & Chile & 1177.8 & & .790 & & & & .059 & .071 & & $\mathrm{X}$ & $\mathrm{X}$ & & & & $\mathrm{X}$ & & $\mathrm{X}$ & $6 \mathrm{~F}$ \\
\hline 3 & Chile & 2569.15 & & .950 & .940 & & & .068 & .060 & $\mathrm{X}$ & & $\mathrm{X}$ & & & & & $\mathrm{x}$ & & $6 \mathrm{~F}$ \\
\hline 4 & México & & 847.05 & .711 & & & & .072 & .080 & & $\mathrm{x}$ & & & $\mathrm{X}$ & & & $\mathrm{x}$ & & $6 \mathrm{~F}$ \\
\hline 5 & Chile & 1100.775 & & .829 & .768 & & .845 & .065 & .062 & & $\mathrm{x}$ & & & $\mathrm{X}$ & & $\mathrm{x}$ & $\mathrm{X}$ & & $6 \mathrm{~F}$ \\
\hline 6 & Argentina & 1224.653 & & .823 & .729 & & .859 & .054 & .076 & & $\mathrm{X}$ & & & $\mathrm{X}$ & & & $\mathrm{X}$ & & $6 \mathrm{~F}$ \\
\hline 7 & Chile & 896.868 & & .814 & .724 & & .811 & .071 & .077 & & $\mathrm{X}$ & & $\mathrm{X}$ & & & $\mathrm{X}$ & $\mathrm{X}$ & & $\begin{array}{c}\text { Mal } \\
\text { ajuste }\end{array}$ \\
\hline 8 & España & 476.29 & & .910 & &. .89 & .93 & .060 & & & $\mathrm{X}^{\mathrm{a}}$ & & & $\mathrm{X}$ & & $\mathrm{X}$ & & $\mathrm{X}$ & $4 \mathrm{~F}$ \\
\hline
\end{tabular}

Nota. 1. Van Dierendonck et al. (2008); 2. Gallardo-Cuadra y Moyano-Díaz (2012); 3. Véliz-Burgos (2012); 4. Medina-Calvillo et al. (2013); 5. VeraVillarroel et al. (2013); 6. Reyna et al. (2013); 7. Chitgian-Urzúa et al. (2013); 8. Freire et al. (2017). NI: No informa; VL: Variables latentes; PO: Puntuaciones observadas; BIF: Bifactor; 2DO: de segundo orden; 6F: modelo de seis factores oblicuos; 1DO: un factor de orden superior; ${ }^{\text {a }}$ solo se informa del modelo reespecificado (17 ítems). 
nacionalidad de los participantes, chilena (Tabla 1). En cuanto a las características del AFC, la matriz de correlación solo ha sido informada en el estudio de Van Dierendonck, Díaz, RodríguezCarvajal, Blanco y Moreno-Jiménez (2008), mientras que el método de estimación más utilizado es el de máxima verosimilitud (37.5\%), además, hay el mismo porcentaje de estudios que no informan el método de estimación (Tabla 1).

La información recopilada sobre los índices de ajuste indica que solo un estudio (VélizBurgos, 2012) presenta valores que pueden ser considerados como aceptables siguiendo los estándares tradicionales para su interpretación $(\mathrm{CFI}>$.95; RMSEA < .06; SRMR < .08; Hu \& Bentler, 1999), y entre los cinco estudios que postulan que el modelo de seis factores es mejor que los competidores, los índices no son adecuados siguiendo las directrices mencionadas $($ CFIpromedio $=.821 ;$ RMSEApromedio $=.064$; SRMRpromedio $=.070)$, mientras que solo los estudios de Chitgian-Urzúa, Urzúa y VeraVillarroel (2013; CFI $=.814$; RMSEA $=.071$; SRMR $=.077)$ y Freire, Ferradas, Núñez y Valle (2017; CFI $=.84$; $\mathrm{RMSEA}=.06)$ evaluaron desfavorablemente el modelo de seis factores con base en sus índices de ajuste (Tabla 1).

Con respecto al reporte de las cargas factoriales, solo dos estudios brindaron esa información de la versión completa de la escala, uno a nivel de ítems (Véliz-Burgos, 2012), y otro de la versión final luego de eliminar ítems (Freire

Tabla 2

Magnitud de las correlaciones interfactoriales (variables latentes) de los modelos oblicuos (AFC).

\begin{tabular}{|c|c|c|c|c|}
\hline & Estudio $1^{\mathrm{a}}$ & Estudio $2^{\text {b }}$ & Estudio $3^{\mathrm{a}}$ & Promedio \\
\hline AA-RP & .59 & .562 & .69 & .614 \\
\hline AA-AU & .58 & .486 & .62 & .562 \\
\hline AA-DO & .89 & .960 & .93 & .927 \\
\hline AA-PR & .97 & .854 & .80 & .875 \\
\hline AA-CR & .74 & .656 & .97 & .789 \\
\hline RP-AU & .38 & .342 & .55 & .424 \\
\hline RP-DO & .59 & .535 & .72 & .615 \\
\hline RP-PR & .63 & .376 & .53 & .512 \\
\hline RP-CR & .49 & .522 & .57 & .527 \\
\hline AU-DO & .58 & .624 & .78 & .661 \\
\hline AU-PR & .62 & .560 & .48 & .553 \\
\hline AU-CR & .48 & .497 & .55 & .509 \\
\hline DO-PR & .96 & .981 & .90 & .947 \\
\hline DO-CR & .74 & .939 & .93 & .87 \\
\hline PR-CR & .79 & .757 & .84 & .796 \\
\hline AA-G & .95 & & & \\
\hline RP-G & .62 & & & \\
\hline AU-G & .61 & & & \\
\hline DO-G & .94 & & & \\
\hline PR-G & 1.00 & & & \\
\hline CR-G & .78 & & & \\
\hline
\end{tabular}

Nota. 1. Van Dierendonck et al. (2008); 2. Gallardo-Cuadra y Moyano-Díaz (2012); 3. Véliz-Burgos (2012); AA: Autoaceptación; RP: Relaciones positivas con los otros; AU: Autonomía; DO: Dominio del entorno; PR: Propósito en la vida; CR: Crecimiento personal; G: Factor general de segundo orden; a: Informaron dos decimales; b: Informaron tres decimales. 
et al., 2017).

Asimismo, solo un $37.5 \%$ de los estudios analizó las correlaciones interfactoriales (CI) con las variables latentes y un estudio analizó estas correlaciones a nivel de las puntuaciones observadas, mientras que el resto no presenta información al respecto. En cuanto a las CI observadas, los valores promedio se encuentran en el rango comprendido entre .424 y .947 (Tabla 2) Otro aspecto relevante es el análisis de los modelos jerárquicos. La PWBS sustenta el uso de una puntuación general sobre la base de un modelo jerárquico indirecto (de segundo orden). Seis estudios (de los nueve que usaron AFC) condujeron dicho análisis (Tabla 1); y de estos, solo uno (Van Dierendonck et al., 2008) reportó los coeficientes de configuración que indican la influencia del factor general sobre los factores específicos (Tabla 2).

Fue realizado un re-análisis de la confiabilidad del constructo evaluada con el coeficiente omega ( $\omega$; McDonald, 1999) con la información brindada en la Tabla 5 de VélizBurgos (2012) y Tabla 5 de Freire et al. (2017). Del total (10), solo cinco coeficientes presentan magnitudes que podrían considerarse como aceptables $(\omega>.70)$.

Tabla 3

Re-análisis de la confiabilidad del constructo.

\begin{tabular}{ccc}
\hline & Estudio 1 & Estudio 2 \\
\hline $\mathrm{AU}$ & .691 & .786 \\
$\mathrm{RP}$ & .745 & \\
$\mathrm{AA}$ & .800 & \\
$\mathrm{DO}$ & .615 & .600 \\
$\mathrm{PR}$ & .784 & .745 \\
$\mathrm{CR}$ & .664 & .690 \\
\hline
\end{tabular}

Nota. 1. Véliz-Burgos (2012); 2. Freire et al. (2017); AA: Autoaceptación; RP: Relaciones positivas con los otros; AU: Autonomía; DO: Dominio del entorno; PR: Propósito en la vida; CR: Crecimiento personal.
Estudios bajo un enfoque exploratorio

El software estadístico utilizado con mayor frecuencia es el SPSS $®$ (IBM Corp.). El 66\% de los estudios realiza análisis preliminares a través del KMO y el Test de Bartlett. En ninguno de los estudios se indica el tipo de matriz utilizada en el AFE, mientras que el método de estimación de máxima verosimilitud fue utilizado en un estudio, el análisis por componentes principales (ACP) fue utilizado en otro y ambos en un tercero. Respecto al criterio para la determinación del número de factores, en un estudio se utilizó el porcentaje de varianza, en otro la regla del autovalor $>1$ y en otro ambos criterios. Las rotaciones utilizadas fueron ortogonales (varimax) en un $66 \%$, solo un estudio reporta rotación oblicua. En dos de los tres estudios se informan las cargas factoriales (Loera-Malvaez, Balcázar-Nava, Trejo-González, Gurrola-Peña, \& Bonilla-Muñoz, 2008; VélizBurgos, 2012; Tabla 4).

Un análisis complementario con los datos disponibles de Loera-Malvaez et al. (2008) y Véliz-Burgos (2012), indica que al comparar los resultados de la regla de autovalor $>1$ con respecto a la cantidad de factores extraídos siguiendo el análisis paralelo (Horn, 1965), es posible detectar una leve sobre-extracción. Dicho análisis fue orientado con la metodología empleada en otro manuscrito (Merino-Soto \& Dominguez-Lara, 2015; Tabla 5)

Por otro lado, fue analizada la simplicidad factorial con los datos presentados en la Tabla 4 de Véliz-Burgos (2012) utilizando el índice de simplicidad factorial (ISF; Coe \& MerinoSoto, 2003), aceptando ISF $\geq .65$ como un criterio flexible para indicar que el ítem está aceptablemente influido por un solo factor. El análisis llevado a cabo indica que solo el $41 \%$ cumple con ese criterio (15 ítems de 37), pero cuando los espacios vacíos (cargas omitidas en 
el reporte de Véliz-Burgos, 2012) se completan con un valor tan bajo como .10 (ya que es poco probable que las cargas en los casilleros sean nulas), el porcentaje se reduce a 27\% (10 ítems de 37, Tabla 6).

\section{Tabla 4a}

Caracterización de los estudios bajo un enfoque factorial exploratorio.

\begin{tabular}{|c|c|c|c|c|c|c|c|c|c|c|c|c|c|}
\hline & & \multicolumn{2}{|c|}{$\begin{array}{c}\text { Grupo etario } \\
\text { (tamaño } \\
\text { muestral) }\end{array}$} & \multicolumn{2}{|c|}{$\begin{array}{l}\text { Programa } \\
\text { estadístico }\end{array}$} & $\begin{array}{c}\text { Matriz de } \\
\text { correlaciones }\end{array}$ & \multicolumn{3}{|c|}{ Análisis preliminares } & \multicolumn{2}{|r|}{ Estimación } & \multicolumn{2}{|c|}{$\begin{array}{c}\text { Criterio para } \\
\text { determinar } \\
\text { número de } \\
\text { factores }\end{array}$} \\
\hline Estudio & País & $\mathrm{AD}$ & UNIV & SPSS & NI & NI & KMO & $\begin{array}{l}\text { Test de } \\
\text { Bartlett }\end{array}$ & NI & ML & $\mathrm{ACP}$ & $\begin{array}{l}\mathrm{V} \text { a } 1 \text { o } \mathrm{r} \\
\text { Eigen }>1\end{array}$ & $\% \mathrm{VE}$ \\
\hline 1 & México & 208 & & $X$ & & $\mathrm{X}$ & & & $\mathrm{X}$ & & $\mathrm{X}$ & $\mathrm{X}$ & \\
\hline 2 & Chile & & 691 & $X$ & & $X$ & $\mathrm{X}$ & $\mathrm{X}$ & & $\mathrm{X}$ & & $X$ & $\mathrm{X}$ \\
\hline 3 & Argentina & & 465 & & $X$ & $X$ & $\mathrm{X}$ & $\mathrm{X}$ & & $\mathrm{X}$ & $\mathrm{X}$ & & $X$ \\
\hline
\end{tabular}

Nota. 1. Loera-Malvaez et al. (2008); 2. Véliz-Burgos (2012); 3. Reyna et al. (2013); AD: Adolescentes; UNIV: Universitarios; NI: No informa; ML: Máxima verosimilitud; ACP: Análisis de componentes principales; \%VE: Porcentaje de varianza explicada.

Tabla 4b

Caracterización de los estudios bajo un enfoque factorial exploratorio.

\begin{tabular}{|c|c|c|c|c|c|c|c|c|c|c|}
\hline & & \multicolumn{2}{|c|}{ Rotación } & \multicolumn{2}{|c|}{$\begin{array}{c}\text { Magnitud } \\
\text { de las cargas } \\
\text { factoriales }\end{array}$} & \multirow[t]{2}{*}{$\begin{array}{c}\text { Magnitud de las } \\
\text { correlaciones }\end{array}$} & \multicolumn{3}{|c|}{ Elimina ítems } & Comentario \\
\hline Estudio & País & ORT & OBL & SÍ & NI & & No & $\begin{array}{c}\text { Sí } \\
\text { (baja carga } \\
\text { factorial) }\end{array}$ & $\begin{array}{c}\text { Sí } \\
\text { (bajo } r_{\text {itc }} \text { ) }\end{array}$ & \\
\hline 1 & México & Varimax & & $\mathrm{X}$ & & No aplica & & $\mathrm{X}$ & & \\
\hline 2 & Chile & & Oblimin & $\mathrm{X}$ & & No informa & & & $\mathrm{X}$ & luego del AFE ejecuta $\mathrm{AFC}$ \\
\hline 3 & Argentina & Varimax & & & $\mathrm{X}$ & No aplica & $\mathrm{X}$ & & & luego del AFE ejecuta AFC \\
\hline
\end{tabular}

Nota. 1. Loera-Malvaez et al. (2008); 2. Véliz-Burgos (2012); 3. Reyna et al. (2013); ORT: Ortogonal; OBL: Oblicua; $\mathrm{r}_{\mathrm{itc}}$ : Correlación ítem-total corregida.

\section{Tabla 5}

Re-análisis de la dimensionalidad en los estudios un enfoque factorial exploratorio.

\begin{tabular}{cccccc}
\hline & \multicolumn{3}{c}{ Estudio 1 } & & \multicolumn{2}{c}{ Estudio 2 } \\
\hline & Valor Eigen real & Valor Eigen aleatorio & & Valor Eigen real & Valor Eigen aleatorio \\
Componente 1 & $\mathbf{1 0 . 7 8 9}$ & 2.045 & Factor 1 & $\mathbf{9 . 6}$ & 1.516 \\
Componente 2 & 2.463 & 1.911 & Factor 2 & $\mathbf{2 . 4}$ & 1.449 \\
Componente 3 & 1.776 & 1.811 & Factor 3 & $\mathbf{1 . 8 4}$ & 1.405 \\
Componente 4 & 1.564 & 1.727 & Factor 4 & $\mathbf{1 . 4 8}$ & 1.365 \\
& & & Factor 5 & 1.31 & 1.331 \\
\end{tabular}

Nota. 1. Loera-Malvaez et al. (2008); 2. Véliz-Burgos (2012). 
Tabla 6

Re-análisis de la simplicidad factorial en Véliz-Burgos (2012).

\begin{tabular}{|c|c|c|c|c|c|c|c|c|}
\hline & F1 & F2 & F3 & F4 & F5 & F6 & ISF $^{\mathbf{a}}$ & ISF $^{t}$ \\
\hline Ítem 1 & .717 & & & -.345 & & .325 & .661 & .623 \\
\hline Ítem 2 & .306 & .392 & .345 & -.595 & & & .444 & .420 \\
\hline Ítem 3 & & & & & .621 & & 1.000 & .862 \\
\hline Ítem 4 & & .596 & & & & & 1.000 & .852 \\
\hline Ítem 5 & .381 & .495 & .498 & -.391 & .314 & & .258 & .246 \\
\hline Ítem 6 & .400 & & .376 & & .404 & .446 & .324 & .296 \\
\hline Ítem 7 & .607 & & .392 & -.369 & .568 & .465 & .274 & .266 \\
\hline Ítem 8 & & & & -.612 & & & 1.000 & .859 \\
\hline Ítem 9 & & .558 & & & & & 1.000 & .834 \\
\hline Ítem 11 & .506 & & & & & .424 & .647 & .566 \\
\hline Ítem 12 & .528 & & .352 & & .418 & .473 & .353 & .331 \\
\hline Ítem 13 & .451 & .444 & .341 & -.320 & .326 & & .252 & .238 \\
\hline Ítem 14 & & & & -.525 & & & 1.000 & .816 \\
\hline Ítem 15 & & .450 & & & .318 & & .683 & .570 \\
\hline Ítem 16 & & & & & & & $\mathrm{NC}$ & $\mathrm{NC}$ \\
\hline Ítem 17 & .752 & & & & .313 & .333 & .696 & .659 \\
\hline Ítem 18 & .657 & & .350 & & .309 & .361 & .499 & .476 \\
\hline Ítem 19 & .567 & & .333 & -.323 & .616 & .383 & .332 & .323 \\
\hline Ítem 20 & .313 & .338 & & -.586 & & & .589 & .541 \\
\hline Ítem 21 & & .317 & & & .545 & & .739 & .647 \\
\hline Ítem 22 & .348 & .435 & .418 & -.383 & & & .329 & .300 \\
\hline Ítem 23 & .515 & & .550 & & .390 & .430 & .348 & .328 \\
\hline Ítem 24 & & & .315 & & .380 & .398 & .463 & .401 \\
\hline Ítem 25 & .536 & .408 & .580 & -.313 & & & .393 & .373 \\
\hline Ítem 26 & & .330 & .363 & -.563 & & & .548 & .502 \\
\hline Ítem 27 & & .409 & .317 & -.327 & .373 & & .336 & .301 \\
\hline Ítem 28 & .388 & .303 & .406 & & .430 & .498 & .249 & .236 \\
\hline Ítem 29 & .302 & & .810 & & .313 & .353 & .626 & .607 \\
\hline Ítem 30 & & & .523 & & & & 1.000 & .815 \\
\hline Ítem 31 & .667 & & .512 & & .425 & .461 & .376 & .360 \\
\hline Ítem 32 & .378 & & & -.418 & & .349 & .457 & .400 \\
\hline Ítem 33 & & .53 & .338 & & & & .712 & .621 \\
\hline Ítem 35 & & & & & & .332 & 1.000 & .626 \\
\hline Ítem 36 & & .326 & .334 & & & & .633 & .486 \\
\hline Ítem 37 & .428 & & & & & .678 & .715 & .657 \\
\hline Ítem 38 & .448 & & .337 & & .349 & .703 & .483 & .464 \\
\hline Ítem 39 & & & & & & .414 & 1.000 & .729 \\
\hline
\end{tabular}

Nota. en negrita: ISF $\geq .65$; ${ }^{a}$ : ISF obtenidos con los datos de Véliz-Burgos (2012); b: ISF obtenidos considerando .10 en los espacios en blanco; NC: No calculado. 


\section{Discusión}

Esta revisión de estudios instrumentales evidencia cómo ha sido estudiada la estructura interna de la versión en español de la PWBS. Si bien en otros espacios se hace mención a algunos problemas en la dimensionalidad de la PWBS (p. ej., Chen et al., 2013; Vera-Villarroel et al. 2013), aquellos resultados no brindan un panorama general sobre la forma en que se han estado conduciendo los estudios instrumentales. Los siguientes párrafos resumen los hallazgos más sobresalientes.

En relación con los análisis basados en el $\mathrm{AFC}$, solo una investigación informó el tipo de correlación utilizada. Una de las fuentes para la elección del tipo de matriz por utilizar es el tipo de respuesta de los ítems (Ferrando \& LorenzoSeva, 2014). Por el tipo de escalamiento ordinal de los ítems de la PWBS (seis opciones de respuesta) se puede asumir que se trata de una variable cuantitativa ( $>5$ opciones; Lozano, García-Cueto, \& Muñiz, 2008), esto puede resultar cuestionable ya que otros aspectos que intervienen son la dispersión y asimetría de los datos, así como las opciones de respuesta efectivas, es decir, las que fueron utilizadas. Cabe recalcar esto ya que la mera existencia de seis opciones no garantiza que todas sean usadas en cantidades aceptables $\mathrm{y}$, por ende, pueden aparecer efectos de techo o piso. Incluso, atendiendo estas características, en ocasiones los escalamientos de cinco o seis opciones suponen medidas ordinales (Bowen \& Masa, 2015; Verdam, Oort, \& Sprangers, 2016). Por ello, es necesario informar aspectos descriptivos de los ítems que permitan conocer su comportamiento de forma más precisa (Malgady, 2007).

En cuanto a las correlaciones interfactoriales promedio encontradas, y pese a su escaso reporte
(37.5\% de los estudios que usaron AFC), se deduce que existe multicolinealidad entre muchas dimensiones $(r>.80)$. Esta situación se asocia con la presencia de complejidad factorial (Graham, Guthrie, \& Thompson, 2003) relacionada con los coeficientes de estructura, así como a potenciales malas especificaciones (Hu \& Bentler, 1998) vinculadas a cargas cruzadas o a correlaciones interfactoriales tan elevadas que harían más congruente considerar, en esencia, un solo factor en lugar de dos (o más), y que dicha estructura multifactorial no sea relevante para la descripción del constructo medido.

Este aspecto lleva a retomar la discusión relacionada con las magnitudes de algunos índices de ajuste informados, ya que aunque los promedios del RMSEA y del SRMR no se alejen significativamente de los estándares mínimos, el RMSEA no es sensible a las cargas cruzadas omitidas (Savalei, 2012) y tiende a mostrar magnitudes más aceptables mientras más indicadores observables (ítems) existan sin importar el grado de mala especificación que posea el modelo (Kenny \& McCoach, 2003), mientras que el CFI se mantiene estable. Asimismo, el SRMR tiende a mostrar valores aceptables incluso en modelos mal especificados basados en una estructura compleja (Marsh, Hau, $\&$ Wen, 2004), como la de la PWBS. A pesar de su importancia, no es conveniente concluir solo con base en los índices de ajuste (Dominguez-Lara, 2016), ya que existen otros aspectos a evaluar en el análisis de la estructura interna (p. ej., malas especificaciones; Hu \& Bentler, 1998).

El análisis jerárquico predominante en la PWBS es el modelo jerárquico indirecto (de segundo orden), cuando lo adecuado es el empleo de un modelo jerárquico directo (bifactor) para sustentar el uso de una puntuación general (Dominguez-Lara \& Rodriguez, 2017), sobre todo en el caso de la PWBS debido a la interpretación 
de una puntuación general postulada por los autores del instrumento, así como a la presencia de multicolinealidad y complejidad factorial. El modelo bifactor capitaliza de forma más precisa la influencia del factor general sobre los ítems mediante distintos indicadores estadísticos (Rodriguez, Reise, \& Haviland, 2016), a diferencia del modelo indirecto que no informa sobre la influencia del factor general $\mathrm{y}$, en consecuencia, no permite derivar conclusiones respecto al uso de una puntuación general (Canivez, 2016).

Como aspecto complementario, la confiabilidad de las puntuaciones informadas en las investigaciones fue estimada mediante el coeficiente alfa de Cronbach. No obstante, no han sido presentados resultados que acrediten el cumplimiento de las condiciones necesarias para su utilización, como por ejemplo el modelo de medición de equivalencia-tau (Dunn, Baguley, \& Brunsden, 2014). Se ha verificado que la estimación del coeficiente alfa de Cronbach bajo violación del supuesto de equivalencia tau genera una subestimación del valor real de la confiabilidad; por este motivo, el coeficiente alfa de Cronbach sería su límite inferior (Eisinga, te Grotenhuis, \& Pelzer, 2013; Raykov, 1997). En este marco, se estimó el coeficiente omega ( $\omega$; McDonald, 1999), el cual es más preciso en supuestos de medición congenéricos (Dunn et al., 2014). De este modo, los coeficientes hallados indicarían que las dimensiones analizadas no reflejan de forma confiable el constructo que evalúan.

Con respecto a los análisis con base en elAFE, aunque fueron solo tres trabajos, se destaca el uso de procedimientos que la literatura psicométrica desestima por limitaciones intrínsecas, como el uso de ACP y la regla del autovalor $>1$ (Ferrando \& Anguiano-Carrasco, 2010; Lloret-Segura, Ferreres-Traver, Hernández-Baeza, \& TomásMarco, 2014); en uno de ellos (Loera-Malvaez et al., 2008) ambos procedimientos coinciden con la rotación varimax, lo que deriva en el paquete conocido como Little Jiffy (Dominguez-Lara \& Merino-Soto, 2016). Por otro lado, dos estudios (Reyna et al., 2013; Véliz-Burgos, 2012) usan de forma consecutiva AFE y AFC con la misma muestra, lo que constituye un procedimiento no recomendado ya que es poco probable que un AFC no valide la solución resultante de AFE en un mismo grupo (Pérez-Gil, Chacón-Moscoso, \& Moreno-Rodríguez, 2000). Finalmente, la ausencia del análisis de la simplicidad factorial priva de una interpretación más pormenorizada sobre el grado de representatividad de los ítems con respecto al constructo que se pretende evaluar.

Los resultados presentados no se alejan de lo observado en otras revisiones respecto a los usos del AF en el análisis de instrumentos de medición. Por ejemplo, en el caso del AFE, el reporte de la matriz de correlaciones es mínimo, el ACP se aplica en más de la mitad de los análisis (58.1\%), y la regla del autovalor $>1$ es la más usada (20.8\%; Izquierdo et al., 2014). Del mismo modo, en cuanto al AFC, el reporte de correlaciones interfactoriales y la ausencia del reporte de cargas factoriales son cercanos al 50\% (Jackson et al., 2009). No obstante, al tratarse de un instrumento en particular (PWBS) se espera cierta uniformidad en cuanto a los procedimientos empleados. Con todo, los re-análisis llevados a cabo brindan evidencia adicional de los potenciales problemas delineados anteriormente, ya que se aprecia que la extracción de seis factores parece no ser necesaria, y la complejidad factorial observada lo confirma. Si a esto se le añade lo concerniente a las elevadas correlaciones interfactoriales, mal ajuste del modelo de seis factores, y los indicadores de confiabilidad del constructo de magnitudes limítrofes, es necesario entonces revisar algunas asunciones básicas del desarrollo teórico del instrumento (Stanley \& Edwards, 
2016), de estructura (p. ej., Padrós-Blásquez, Herrera-Guzmán, \& Gudayol-Ferré, 2012) o, en su defecto, derivar versiones breves pero estructuralmente más robustas (p. ej., mediante la selección de ítems con mayor identificación con el factor específico que con el FG), que permitan evaluar de forma válida las dimensiones que postula Ryff.

En conclusión, se observa que algunas prácticas no contribuyen a una lectura completa de los resultados (p. ej., omisión de la información sobre cargas factoriales), perpetúan el uso de procedimientos no recomendados (p. ej., uso del Little Jiffy) o no brindan información suficiente para el manejo de las puntuaciones totales (p. ej., ausencia de información sobre modelos bifactor). A su vez, un aspecto a destacar es el estudio del efecto del método asociado a los ítems invertidos que no fue informado en ninguno de los estudios revisados, aunque sí se observa en otras versiones de la PWBS (Tomas, Meléndez, Oliver, Navarro, \& Zaragoza, 2010), y resulta importante conocer el impacto del fraseo de los ítems. Por estos motivos, aún no existiría claridad respecto a la estructura interna de la PWBS-E. Cabe precisar que una limitación del estudio es que no se realizó la búsqueda en bases de datos como PsycInfo y Web of Science, en las que se encuentran revistas especializadas y de alto impacto a la comunidad científico psicológica, debido a que no se contó con acceso a las mismas.

Por último, cabe resaltar que el presente estudio es el primero en Sudamérica orientado a la revisión de estudios psicométricos sobre la dimensionalidad de un instrumento específico. En otras latitudes se han realizado estudios de revisión sistemática y se han obtenido resultados interesantes, aunque abordando tópicos generales (p. ej., Alberts, Hadjistavropoulos, Jones, \& Sharpe, 2013). En tal sentido, se recomienda implementar esta práctica con otros instrumentos que, en su momento, han sido reportados como los de mayor uso en Latinoamérica (Prieto, Muñiz, Almeida, \& Bartram, 1999), sobre todo con aquellos usados para toma de decisiones en contextos profesionales.

\section{Referencias}

Abbott, R. A., Ploubidis, G. B., Huppert, F. A., Kuh, D., \& Croudace, T. J. (2010). An evaluation of the precision of measurement of Ryff's Psychological Well-Being Scales in a population sample. Social Indicators Research, 97(3), 357-373. doi: 10.1007/ s11205-009-9506-x

Alberts, N. M., Hadjistavropoulos, H. D., Jones, S. L., \& Sharpe, D. (2013). The Short Health Anxiety Inventory: A systematic review and meta-analysis. Journal of Anxiety Disorders, 27(1), 68-78. doi: 10.1016/j.janxdis.2012.10.009

American Educational Research Association, American Psychological Association, \& National Council on Measurement in Education (2014). Standards for Educational and Psychological Testing. Washington, DC: American Educational Research Association.

Ato, M., López, J. J., \& Benavente, A. (2013). Un sistema de clasificación de los diseños de investigación en psicología. Anales de Psicología, 29(3), 1038-1059. doi: 10.6018/analesps.29.3.178511

Bowen, N. K., \& Masa, R. D. (2015). Conducting measurement invariance tests with ordinal data: A guide for social work researchers. Journal of the Society for Social Work and Research, 6(2), 229-249. doi: 10.1086/681607

Canivez, G. L. (2016). Bifactor modeling in construct validation of multifactored tests: Implications for multidimensionality and test interpretation. En K. Schweizer \& C. DiStefano (Eds.), Principles and methods of test construction: Standards and recent advancements (pp. 247-271). Gottingen, Germany: Hogrefe. doi: 10.1027/00449-000 
Chen, F. F., Jing, Y., Hayes, A., \& Lee, J. M. (2013). Two concepts or two approaches? A bifactor analysis of psychological and subjective well-being. Journal of Happiness Studies, 14(3), 1033-1068. doi: 10.1007/ s10902-012-9367-X

Chitgian-Urzúa, V., Urzúa, A., \& Vera-Villarroel, P. (2013). Análisis preliminar de las Escalas de Bienestar Psicológico en población chilena. Revista Argentina de Clínica Psicológica, 22(1), 5-14. Recuperado de http://www.clinicapsicologica.org.ar

Coe, R., \& Merino-Soto, C. (2003). Medidas de simplicidad y ajuste factorial: Un enfoque para la construcción y revisión de escalas derivadas factorialmente. Revista de Psicología, 21(1), 252-266. Recuperado de http:// revistas.pucp.edu.pe/index.php/psicologia/article/ view/3722

Díaz, D., Rodríguez-Carvajal, R., Blanco, A., MorenoJiménez, B., Gallardo, I., Valle, C., \& Van Dierendonck, D. (2006). Adaptación española de las Escalas de Bienestar Psicológico de Ryff. Psicothema, 18(3), 572-577. Recuperado de http:// www.psicothema.com

Dominguez-Lara, S. A. (2016). Evaluación de modelos estructurales, más allá de los índices de ajuste. Enfermería Intensiva, 27(2), 84-85. doi: 10.1016/j. enfi.2016.03.003

Dominguez-Lara, S. A., \& Merino-Soto, C. (2016). Sobre o uso do Little Jiffy na validação dos testes: Comentários a Ávila e colaboradores. Jornal Brasileiro de Psiquiatria, 65(2), 196-197. doi: 10.1590/0047-2085000000123

Dominguez-Lara, S. A., \& Rodriguez, A. (2017). Índices estadísticos de modelos bifactor. Interacciones. Revista de Avances en Psicología, 3(2), 59-65. doi: 10.24016/2017.v3n2.51

Dunn, T. J., Baguley, T., \& Brunsden, V. (2014). From alpha to omega: A practical solution to the pervasive problem of internal consistency estimation. British Journal of Psychology, 105(3), 399-412. doi: 10.1111/bjop.12046

Eisinga, R., Te Grotenhuis, M., \& Pelzer, B. (2013). The reliability of a two-item scale: Pearson, Cronbach, or Spearman-Brown. International Journal of Public Health, 58(4), 637-642. doi: 10.1007/s00038-0120416-3

Ferrando, P. J., \& Anguiano-Carrasco, C. (2010). El análisis factorial como técnica de investigación en psicología. Papeles del Psicólogo, 31(1), 18-33. Recuperado de www.papelesdelpsicologo.es

Ferrando, P. J., \& Lorenzo-Seva, U. (2014). El análisis factorial exploratorio de los ítems: Algunas consideraciones adicionales. Anales de Psicología, 30(3), 1170-1175. doi: 10.6018/analesps.30.3.199991

Freire, C., Ferradás, M. del M., Núñez, J. C., \& Valle, A. (2017). Estructura factorial de las Escalas de Bienestar Psicológico de Ryff en estudiantes universitarios. European Journal of Education and Psychology, 10(1), 1-8. doi: 10.1016/j.ejeps.2016.10.001

Gallardo-Cuadra, I., \& Moyano-Díaz, E. (2012). Análisis psicométrico de las escalas Ryff (versión española) en una muestra de adolescentes chilenos. Universitas Psychologica, 11(3), 931-939. Recuperado de http:// revistas.javeriana.edu.co/index.php/revPsycho/ index/

Graham, J. M., Guthrie, A. C., \& Thompson, B. (2003). Consequences of not interpreting structure coefficients in published CFA research: A reminder. Structural Equation Modeling: A Multidisciplinary Journal, 10(1), 142-153. doi: 10.1207/ S15328007SEM1001_7

Horn, J. L. (1965). A rationale and test for the number of factors in factor analysis. Psychometrika, 30(2), 179185. doi: 10.1007/BF02289447

Hu, L., \& Bentler, P. M. (1998). Fit indices in covariance structure modeling: Sensitivity to underparameterized model misspecification. Psychological Methods, 3(4), 424-453. doi: 10.1037/1082-989X.3.4.424

Hu, L., \& Bentler, P. M. (1999). Cutoff criteria for fit indexes in covariance structure analysis: Conventional criteria versus new alternatives. Structural Equation Modeling: A Multidisciplinary Journal, 6(1), 1-55. doi: 10.1080/10705519909540118 
IBM Corp. IBM SPSS Statistics for Windows [software de cómputo]. Armonk, NY: IBM Corp.

Izquierdo, I., Olea, J., \& Abad, F. J. (2014). Exploratory factor analysis in validation studies: Uses and recommendations. Psicothema, 26(3), 395-400. Recuperado de http:/www.psicothema.com/

Jackson, D. L., Gillaspy, J. A., \& Purc-Stephenson, R. (2009). Reporting practices in confirmatory factor analysis: An overview and some recommendations. Psychological Methods, 14(1), 6-23. doi: 10.1037/ a0014694

Kenny, D. A., \& McCoach, D. B. (2003). Effect of the number of variables on measures of fit in structural equation modeling. Structural Equation Modeling: A Multidisciplinary Journal, 10(3), 333-351. doi: 10.1207/S15328007SEM1003_1

Lloret-Segura, S., Ferreres-Traver, A., Hernández-Baeza, A., \& Tomás-Marco, I. (2014). El análisis factorial exploratorio de los ítems: Una guía práctica, revisada y actualizada. Anales de Psicología, 30(3), 11511169. doi: 10.6018/analesps.30.3.199361

Loera-Malvaez, N., Balcázar-Nava, P., Trejo-González, L., Gurrola-Peña, G. M., \& Bonilla-Muñoz, M. P. (2008). Adaptación de la Escala de Bienestar Psicológico de Ryff en adolescentes preuniversitarios. Revista Neurología, Neurocirugía y Psiquiatría, 41(3-4), 90-97. Recuperado de http://www.medigraphic.com/ cgi-bin/new/publicaciones.cgi?IDREVISTA $=96$

Lozano, L. M., García-Cueto, E., \& Muñiz, J. (2008). Effect of the number of response categories on the reliability and validity of rating scales. Methodology. European Journal of Research Methods for the Behavioral and Social Sciences, 4(2), 73-79. doi: 10.1027/16142241.4.2.73

Malgady, R. G. (2007). How skewed are psychological data? A standardized index of effect size. The Journal of General Psychology, 134(3), 355-359. doi: 10.3200/ GENP.134.3.355-360

Marsh, H. W., Hau, K. T., \& Wen, Z. (2004). In search of golden rules: Comment on hypothesis-testing approaches to setting cutoff values for fit indexes and dangers in overgeneralizing $\mathrm{Hu}$ and Bentler's (1999) findings. Structural Equation Modeling: A Multidisciplinary Journal, 11(3), 320-341. doi: 10.1207/s15328007sem1103_2

McDonald, R. P. (1999). Test theory: A unified treatment. Mahwah, N.J.: L. Erlbaum Associates.

Medina-Calvillo, M. A., Gutiérrez-Hernández, C. Y., \& Padrós-Blázquez, F. (2013). Propiedades psicométricas de la Escala de Bienestar Psicológico de Ryff en población mexicana. Revista Educación Desarrollo, 27, 25-30. Recuperado de http://www. cucs.udg.mx/revistas/edu_desarrollo/index.php

Merino-Soto, C., \& Domínguez-Lara, S. (2015). Sobre la elección del número de factores en estudios psicométricos en la Revista Latinoamericana de Ciencias Sociales, Niñez y Juventud. Revista Latinoamericana de Ciencias Sociales, Niñez y Juventud, 13(2), 1320-1322. Recuperado de http:// revistaumanizales.cinde.org.co/

Mokkink, L. B., Terwee, C. B., Patrick, D. L., Alonso, J., Stratford, P. W., Knol, D. L. ... de Vet, H. C. W. (2012). The COSMIN Checklist Manual. Recuperado de http://www.cosmin.nl

Padrós-Blásquez, F., Herrera-Guzmán, I., \& GudayolFerré, E. (2012). Propiedades psicométricas de la Escala de Gaudibilidad en una población mexicana. Revista Evaluar, 12(1), 1-20. Recuperado de https:// revistas.unc.edu.ar/index.php/revaluar/

Pérez-Gil, J. A., Chacón-Moscoso, S., \& MorenoRodríguez, R. (2000). Validez de constructo: El uso de análisis factorial exploratorio-confirmatorio para obtener evidencias de validez. Psicothema, 12(Suplemento 2), 442-446. Recuperado de http:// www.psicothema.com

Pérez-Verduzco, G., \& Laca-Arocena, F. A. (2017). Traducción y validación de la versión abreviada del Freiburg Mindfulness Inventory (FMI-14). Revista Evaluar, 17(1), 80-93. Recuperado de https://revistas. unc.edu.ar/index.php/revaluar/

Prieto, G., Muñiz, J., Almeida, L. S., \& Bartram, D. (1999). Uso de los tests psicológicos en España, 
Portugal e Iberoamérica. Revista Iberoamericana de Diagnóstico y Evaluación Psicológica, 8(2), 67-82. Recuperado de http://www.aidep.org

Raykov, T. (1997). Estimation of composite reliability for congeneric measures. Applied Psychological Measurement, 21(2), 173-184. doi: $10.1177 / 01466216970212006$

Reise, S. P. (2012). The rediscovery of bifactor measurement models. Multivariate Behavioral Research, 47(5), 667-696. doi: 10.1080/00273171.2012.715555

Reyna, G. V., Delfino, G. I., \& Mele, S. V. (Noviembre, 2013). Estudio psicométrico de la Escala de Bienestar Psicológico en militares argentinos. Ponencia presentada en el $V$ Congreso Internacional de Investigación y Práctica Profesional en Psicología. XX Jornadas de Investigación. Noveno Encuentro de Investigadores en Psicología del MERCOSUR. Facultad de Psicología de la Universidad de Buenos Aires.

Rios, J., \& Wells, C. (2014). Validity evidence based on internal structure. Psicothema, 26(1), 108-116. Recuperado de http://www.psicothema.com/

Rodriguez, A., Reise, S. P., \& Haviland, M. G. (2016). Evaluating bifactor models: Calculating and interpreting statistical indices. Psychological Methods, 21(2), 137-150. doi: 10.1037/met0000045

Ryff, C. D. (1989). Happiness is everything, or is it? Explorations on the meaning of psychological well-being. Journal of Personality and Social Psychology, 57(6), 1069-1081. doi: 10.1037/00223514.57.6.1069

Ryff, C. D. (1995). Psychological well-being in adult life. Current Directions in Psychological Science, 4(4), 99-104. doi: 10.1111/1467-8721.ep10772395

Ryff, C. D., \& Keyes, C. L. M. (1995). The structure of psychological well-being revisited. Journal of Personality and Social Psychology, 69(4), 719-727. doi: 10.1037/0022-3514.69.4.719

Savalei, V.(2012). Therelationship between rootmean square error of approximation and model misspecification in confirmatory factor analysis models. Educational and Psychological Measurement, 72(6), 910-932. doi: $10.1177 / 0013164412452564$

Stanley, L. M., \& Edwards, M. C. (2016). Reliability and model fit. Educational and Psychological Measurement, 76(6), 976-985. doi: 10.1177/0013164416638900

Tomas, J. M., Meléndez, J. C., Oliver, A., Navarro, E., \& Zaragoza, G. (2010). Efectos de método en las escalas de Ryff: Un estudio en población de personas mayores. Psicológica, 31(2), 383-400. Recuperado de https://www.uv.es/revispsi

Triadó, C., Villar, F., Solé, C., \& Celdrán, M. (2007). Construct validity of Ryff's Scale of Psychological Well-being in Spanish older adults. Psychological Reports, 100(3), 1151-1164. doi: 10.2466/ pr0.100.4.1151-1164

Villar, F., Osuna, M. J., Solé, C., \& Triadó, C. (2005). La medida del bienestar en personas mayores: Adaptación de la Escala Ryff. Revista de Psicología General y Aplicada, 58(3), 347-363.

Van Dierendonck, D., Díaz, D., Rodríguez-Carvajal, R., Blanco, A., \& Moreno-Jiménez, B. (2008). Ryff's six-factor model of psychological well-being, a Spanish exploration. Social Indicators Research, 87(3), 473-479. doi: 10.1007/s11205-007-9174-7

Véliz-Burgos, A. (2012). Propiedades psicométricas de la Escala de Bienestar Psicológico y su estructura factorial en universitarios chilenos. Psicoperspectivas. Individuo y Sociedad, 11(2), 143163. doi: 10.5027/psicoperspectivas-Vol11-Issue2fulltext-196

Vera-Villarroel, P., Urzúa M., A., Silva, J. R., Pavez, P., \& Celis-Atenas, K. (2013). Escala de Bienestar de Ryff: Análisis comparativo de los modelos teóricos en distintos grupos de edad. Psicologia: Reflexão e Crítica, 26(1), 106-112. doi: 10.1590/S010279722013000100012

Verdam, M. G., Oort, F. J., \& Sprangers, M. A. G. (2016). Using structural equation modeling to detect response shifts and true change in discrete variables: An application to the items of the SF-36. Quality of Life Research, 25(6), 1361-1383. doi: 10.1007/ s11136-015-1195-0 